\title{
RECHERCHES SUR LES RELATIONS ENTRE L'EUF ET LE TRACTUS MATERNEL PENDANT LES PREMIERS STADES DU DÉVELOPPEMENT CHEZ LES MAMMIFÈRES ÉTUDE DE LA TRAVERSÉE DE L'OVIDUCTE PAR L'CEUF FÉCONDÉ DE BREBIS
}

\begin{abstract}
PAR
Suzanne WINTENBERGER

Station de Physiologie Animale, Centre National de Recherches Zootechniques, Jouy-en-Josas (S.-et-O.)
\end{abstract}

Des opinions contradictoires ont été émises concernant les relations qui existent entre l'œuf fécondé an cours de sa migration tubaire et l'oviducte. Pour ALDEN, Pincus, le développement de l'œuf ne nécessite aucun milieu tubaire spécial; WestMan, Washburn, ont abouti à des conclusions opposées.

Pourtant ce sont les mécanismes mis en jeu qui conditionnent le succès du développement et de l'implantation ultérieure des œufs présents naturellement ou artificiellement dans l'oviducte d'une femelle. Pour étudier cette interaction cuf-oviducte les auteurs précédents ont emprisonné les oufs dans une partie de la trompe. Avant d'employer cette technique il nous est apparu nécessaire de préciser, chez la Brebis, dans des conditions naturelles, à des temps variés après la ponte, à quel niveau du tractus femelle se trouvent le oul les œufs, en observant chaque fois le progrès de leur segmentation.

Une série d'observations a porté sur 24 Brebis de race Bizet et 2 Brebis Lacaune. Elle reçurent dès 1'apparition des chaleurs, une injection intraveineuse de $800 \mathrm{U}$. I. de sérum de jument gravide afin de provoquer l'ovulation $24 \mathrm{~h}$ plus tard (OrTavant, 'Thibault et Wintenderger, I949) et furent ensuite accouplées.

Immédiatement après l'abatage, l'ampoule et 1'isthme furent perfusés par segments et l'utérus en totalité. Il fut toujours admis que l'œuf se trouvait au milieu du segment d'oviducte qui le contenait.

On constate que, pendant les premières heures, 1 'œuf descend rapidement: $2 \mathrm{~h}$ à $2 \mathrm{~h} 30$ après l'ovulation, on le retrouve vers le milieu ou le tiers inférieur de l'ampoule; mais il faut compter 6 à $8 \mathrm{~h}$ pour qu'il atteigne la partie inférieure de celle-ci ( 7 Brebis). Il y séjourne alors jusqu'à 1a $44^{\mathrm{e}} \mathrm{h}$ (8 Brebis). 
TABLEAU I

\begin{tabular}{|c|c|c|c|c|}
\hline \multirow{2}{*}{$\begin{array}{c}\text { Temps } \\
\text { en heures }\end{array}$} & \multicolumn{2}{|c|}{ Ampoule } & \multirow{2}{*}{$\begin{array}{l}\text { Isthme } \\
\text { (Début) }\end{array}$} & \multirow{2}{*}{ Utérus } \\
\hline & I $/ 3$ moyen & $\mathrm{I} / 3$ inférieur & & \\
\hline $\begin{array}{c}\mathbf{2} \\
2,30 \\
6 \\
8 \\
24 \text { à } 44,30 \\
49 \text { à } 56 \\
72 \text { à } 80\end{array}$ & I Brebis & $\begin{array}{ll}\text { I } & \text { Brebis } \\
4 & \text { Brebis } \\
3 & \text { Brebis } \\
8 & \text { Brebis }\end{array}$ & 3 l3rebis & 4 Brebis \\
\hline
\end{tabular}

De 49 à $56 \mathrm{~h}$ après la ponte, l'œuf s'engage dans l'isthme, il est situé alors dans les premiers centimètres de celui-ci (3 Brebis).

Enfin $72 \mathrm{~h}$ après l'ovulation, comme on avait coutume de l'admettre (Assheton, I899 ; Thibault, I949 ; Robinson, I950) l'œuf atteint 1'utérus. La traversée de 1 'isthme est donc relativement rapide.

Cependant nous avons rencontré quelques cas de progression différente, qui nous permettent de tirer des conclusions intéressantes sur les rapports œuf-oviducte.

Ainsi chez une Brebis, l'œuf se trouvait déjà dans la partie supérieure de l'isthme $26 \mathrm{~h}$ après l'ovulation (1'ampoule était relativement très courte, $7 \mathrm{~cm}$ contre II, $6 \mathrm{~cm}$ de longueur moyenne). Par contre, chez 2 Brebis, des œufs séjournaient encore dans le milieu de l'ampoule, respectivement $5 \mathrm{I}$ h et $73 \mathrm{~h}$ après l'ovulation.

Ces variations dans la vitesse de progression de l'œuf n'affectent pas sa vitesse de segmentation. En effet, le tableau II nous montre que les œufs sont toujours aux stades 2 et 4 , respectivement 24 et $44 \mathrm{~h}$ après 1'ovulation. Vers $48 \mathrm{~h}$ ils commencent à se diviser en 8 pour atteindre le stade 8-I2 au bout de $72 \mathrm{~h}$.

TABLEAU II

\begin{tabular}{|c|c|c|c|c|}
\hline \multirow{2}{*}{$\begin{array}{l}\text { Numéro } \\
\text { des Brebis }\end{array}$} & \multirow{2}{*}{$\begin{array}{c}\text { Temps } \\
\text { en heures }\end{array}$} & \multicolumn{3}{|c|}{ Nombre de blastomères } \\
\hline & & $\begin{array}{l}\text { Ampoule } \\
\mathrm{I} / 3 \text { inférieur }\end{array}$ & $\begin{array}{l}\text { Isthme } \\
\text { I } 2 \text { supérieur }\end{array}$ & Utêrus \\
\hline $\begin{array}{r}\mathrm{I} 2 \\
45 \mathrm{I} \\
58 \\
7 \\
44 \\
\mathrm{r} \\
62 \\
27 \\
63\end{array}$ & $\begin{array}{c}24 \\
26 \\
30 \\
44,30 \\
49 \\
73 \\
73 \\
72,30 \\
80\end{array}$ & $\begin{array}{r}2 \\
2 \\
4 \\
8 \\
12\end{array}$ & 8 & 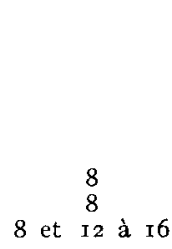 \\
\hline
\end{tabular}


Nous avons pu confirmer expérimentalement cette indépendance entre l'état de la segmentation de 1'œuf et la place qu'il occupe, par une série d'expériences portant sur I3 Brebis chez lesquelles nous avons déclenché une superovulation importante en injectant $4000 \mathrm{U}$. I. de

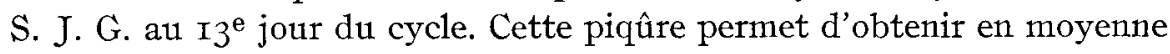
8,8 ovulations par Brebis. (DAuzIER, ThibaulT et Wintenberger, I953).

Dans ces conditions les œufs ont toujours atteint la partie inférieure de l'ampoule $2 \mathrm{~h}$ après l'ovulation et commencent dans certains cas, à s'engager dans l'isthme au bout de $\mathrm{I} 8 \mathrm{~h}$. On peut admettre que, de $\mathrm{I} 8 \mathrm{à}$ $26 \mathrm{~h}$ au lieu de 49 à $56 \mathrm{~h}$ pour les ovulations simples, les œufs s'engagent dans l'isthme, tandis que $48 \mathrm{~h}$ après, ils sont déjà dans l'utérus.

TABLEAU III

\begin{tabular}{|c|c|c|c|c|}
\hline \multirow{2}{*}{$\begin{array}{l}\text { Temps } \\
\text { en heures }\end{array}$} & \multicolumn{2}{|c|}{ Ampoule } & \multirow{2}{*}{$\begin{array}{l}\text { Isthme } \\
\text { (début) }\end{array}$} & \multirow{2}{*}{ Utérus } \\
\hline & I /3 moyen & $\mathrm{I} / 3$ inférieıır & & \\
\hline $\begin{array}{c}2 \text { à } 6 \\
\\
I 6 \\
I 7 \cdot 30 \\
18 \\
25 \cdot 30 \\
26 \\
27 \\
32 \\
47 \\
48\end{array}$ & $\begin{array}{ll}N^{0} & 54 \\
N^{0} & 49 \\
& \\
N^{0} & 38 \\
N^{0} & 2\end{array}$ & $\begin{array}{ll}\text { No } & 35 \\
\text { No } & 45 \\
\text { No } & 67 \\
\text { No } & 54 \\
\text { No } & 48 \\
\text { No } & 49 \\
& \\
\text { No } & 43 \\
\text { No } & 38\end{array}$ & $\begin{array}{ll}\text { No } & 48 \\
\text { No } & 49 \\
\text { No } & 46 \\
\text { No } & 43 \\
& 43 \\
\text { No } & \text { 19 } \\
\text { No } & 2\end{array}$ & $\begin{array}{ll}\text { No } & 48 \\
\text { No } & 43 \\
\text { No } & \\
\text { No } & 2 \\
\text { No } & 464 \\
\text { No } & 459\end{array}$ \\
\hline
\end{tabular}

Les œufs portent les numéros de la Brebis chez laquélle ils ont été retrouvés.

En dépit de cette progression accélérée, l'œuf reste sensiblement fidèle au rythme de développement déjà défini dans le cas d'une seule ovulation : deux cellules au bout de $24 \mathrm{~h}, 3$ à 4 cellules de 26 à $32 \mathrm{~h}, 8$ cellules, $48 \mathrm{~h}$ après son émission.

Tableav IV

\begin{tabular}{|c|c|c|c|c|}
\hline \multirow[b]{2}{*}{ Numéro } & \multirow{2}{*}{$\begin{array}{l}\text { Temps } \\
\text { en heures }\end{array}$} & \multicolumn{3}{|c|}{ Nombre de Blastomères } \\
\hline & & $\begin{array}{l}\text { Ampoule } \\
\text { I } 3 \text { inférieur }\end{array}$ & $\begin{array}{l}\text { Isthme } \\
\text { (début) }\end{array}$ & Utérus \\
\hline 48 & 17,30 & \multirow{4}{*}{\multicolumn{2}{|c|}{ 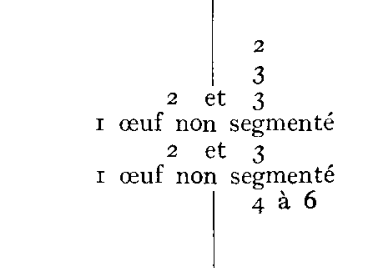 }} & 4 \\
\hline $\begin{array}{l}46 \\
43\end{array}$ & $\begin{array}{c}25,30 \\
26\end{array}$ & & & 2 à 4 \\
\hline $3^{8}$ & 27 & & & \\
\hline $\begin{array}{c}19 \\
464 \\
459\end{array}$ & $\begin{array}{l}32 \\
48 \\
48\end{array}$ & & & $\begin{array}{r}4 \text { à } 5 \\
8 \\
8 \text { à }\end{array}$ \\
\hline
\end{tabular}


Ainsi chez la Brebis, en provoquant l'émission de l'œuf à un instant connu, nous avons pu suivre avec rigueur sa vitesse de déplacement et son développpement : 1'œuf progresse très rapidement dans l'ampoule (vitesse moyenne, I, $4 \mathrm{~cm}$ par heure) mais séjourne dans sa partie inférieure de 6 à $56 \mathrm{~h}$; il commence à pénétrer dans l'isthme à partir de $48 \mathrm{~h}$ et arrive dans l'utérus $72 \mathrm{~h}$ environ, après l'ovulation.

Le long séjour de l'ouf dans la partie inférieure de l'ampoule permettrait de penser que le milieu rencontré est nécessaire à la suite du développement. Mais nous avons vu que la vitesse de transfert de l'œuf peut varier alors que son rythme de division reste constant, tel est le cas, en particulier des œufs " superovulés " dont la descente est accélérée tandis que la vitesse de segmentation demeure invariable. I1 y a donc chez 1a Brebis une indépendance totale entre les premières divisions de segmentation de 1'œuf et la place qu'il occupe dans l'oviducte.

On peut donc penser que, pendant. les premiers stades de son déveveloppement, l'œuf trouve dans tout le tractus les éléments nécessaires, ou qu'il n'est pas tributaire du milieu maternel. Les résultats de $A_{L}, \mathrm{DE}, \mathrm{N}$ montrant que l'œuf de Rate poursuit son développement normal après ovariectomie, parlent en faveur de cette dernière hypothèse. Cependant 1'échec des tentatives mêmes récentes (WASHBURN Jr, I95 I) pour cultiver in vitro l'œuf de Rate, laisse plutôt supposer qu'il existe dans le milieu tubaire des facteurs nécessaires à sa division. Le problème apparait donc comme très complexe. D'ailleurs même lorsque la culture de l'œuf se révèle possible in vitro pendant plusieurs jours comme chez la Lapine, les expériences de transplantation de CHANG, ont bien mis en évidence qu'il ne peut y avoir plus de $24 \mathrm{~h}$ de différence entre l'âge de l'œuf et l'âge pseudogravide du tractus hôte, pour que le développement se poursuive normalement.

Il est donc impossible de conclure à l'indépendance totale de 1'œuf par rapport au milieu maternel, même pendant les premiers stades. Il est d'ailleurs probable que l'apport du milieu maternel devient de plus en plus important au fur et à mesure que les réserves vitellines s'épuisent, aussi doit-il exister de grandes variations dans les relations œuf-oviducte selon les espèces, puisque la vitesse de segmentation propre de l'œuf est plus ou moins rapide : celui de Brebis atteint 1'utérus $72 \mathrm{~h}$ après la ponte au stade 8-12 blastomères, tandis que ceux de Rate ou de Lapine, qui parcourent l'oviducte également en trois jours contiennent une centaine de cellules quand ils atteignent 1'utérus.

Seules des cultures in vitro dans des milieux variés et des ligatures prolongées à des niveaux différents du tractus permettront de définir les besoins de l'œuf et le rôle des différentes parties du tractus femelle dans son développement. 


\section{BIBLIOGRAPHIE}

Alden (R. H.). - Anat. Rec., I942 a, 84, I37-I69.

ALDEN (R. H.). - J. Exp. Zool., I942 b, 90, I59-170.

AlDEN (R. H.). - J. Exp. Zool., I942 c, 90, I7I-I82.

Assheton (R.). - Quarterl. J. Microsc. Sci., I899, 41, 205-262.

Chang (M. C.).-J. Exp. Zool., I950, 114, I97-225.

Dauzier (L.), Thibault (C.), Wintenberger (S.). - Ann. Zootechnie, I953, sous presse.

Ortavant (R.), Thibault (C.), Wintenberger (S.). - Ann. Endoc., I949, $10, n^{\circ} 2$, I $70-I 73$.

Prncus (G.). - "The eggs of Mammals ", I936, I vol.

Robinson ('T. J.). - J. A gric. Sci., I950, 41, 6-63.

Thibault (C.). - Ann. Sc. Nat. Zool., I949, I I érie, XI, I36-2 I9.

Washburn (W. W. Jr.). - Arch. Biol., I95I, 62, 439-458.

Westman (A.). - Acta Obst. Gynec. Scand., I930, 10, 288-298.

I.N.R.A.

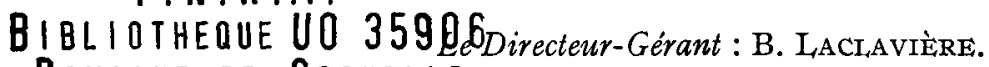

DOMAINE DE CROUELLE

63039

CLERMONT-FD CEDEX 2 